Original Research Paper

\title{
Biofidel FEA Modeling of Customized Hybrid Biological Hip Joint Design Part II: Flexible Stem Trabecular Prostheses
}

\author{
${ }^{1}$ Raffaella Aversa, ${ }^{2}$ Florian Ion T. Petrescu, ${ }^{3}$ Relly Victoria V. Petrescu and ${ }^{1}$ Antonio Apicella \\ ${ }^{1}$ Advanced Materials Lab, Dept of Architecture and Industrial Design, Second University of Naples, Aversa (CE) Italy \\ ${ }^{2}$ Department of Theory of Mechanisms and Robots, Bucharest Polytechnic University, Bucharest, Romania \\ ${ }^{3}$ Department of Transport, Traffic and Logistics, Bucharest Polytechnic University, Bucharest, Romania
}

Article history

Received: 07-10-2016

Revised: $15-12-2016$

Accepted: 16-12-2016

Corresponding Author:

Florian Ion T. Petrescu

Department of Theory of

Mechanisms and Robots,

Bucharest Polytechnic

University, Bucharest, Romania

Email: scipub02@gmail.com

\begin{abstract}
New biomechanical criteria for the design of biomimetic hip joint prostheses are presented. Biofidel Finite Element Models have been used both to correctly analyze femur physiological and structural behavior and to assess a new design criterion for the development of innovative hybrid biological hip prostheses. Proper identification of isostatic lines and isorigidity regions for the sintered metal trabecular lattices distribution between the proximal and distal part of the stem has been carried out. The faithful models enables us to properly take into account not-isotropic properties of the femur proximal end, while clarifying the critical mechanical role of the trabecular bone that should be taken into account to design new innovative prosthetic system.
\end{abstract}

Keywords: Trabecular Lattice, Biomimetic, Biomechanics, Trabecular Prostheses

\section{Introduction}

The validation of the clinical efficiency and the estimate the long-term reliability of prosthetic restorative systems need the appropriate understanding of the physical variables that influence the biomechanical behavior of the material for biomedical advanced applications.

The tool of the Finite Element Analysis (FEA) is allowing biomaterials researchers to attain comprehensive evaluation of the biologic and mechanical behaviors of advanced restorative systems, even in the case of not homogeneous systems.

If validated by proper experimental procedures, the FEA turns useful in the optimization of the restorative design criteria and in the choice of the materials to be used. Moreover, this method allows the estimate of the location of fractures under given loading circumstances (Aversa et al., 2016; Mullender and Huiskes, 1995).

New fabrication processes based on additive manufacturing technologies and studies on biomechanics and biomimetics (Annunziata et al., 2006; Apicella et al., 2010; Aversa et al., 2009) could enable the set-up of new design criteria for human prostheses. The Authors have taken up these studies to gathering the unexploited potential of such advanced materials and design technologies by developing biofidel Finite Element models able to correctly mimic the femur biomechanical behavior (Fig. 1).
Although human femur has an internal structure that, through the evolutionary optimization of the mass and disposition of the cortical and trabecular bone types (Gottesman and Hashin, 1980; Oh and Harris, 1976), is able to bear high external stresses (Ashman et al., 1984; Dalstyra et al., 1993). The progressive physiological bone mass loss occurring in elderly people or in presence of prolonged inactivity, leads to the bone toughness weakening and to a reduction of its ability to resist to mechanical shocks. This reduced resilience is the main cause of elder people pertrochanteric femur fracture (Ashman and Rho, 1988; Burnstein et al., 1976; Carter and Hayes, 1977). The reduction of these fractures and recovery of the hip joint functionality needs the application of special prosthesis.
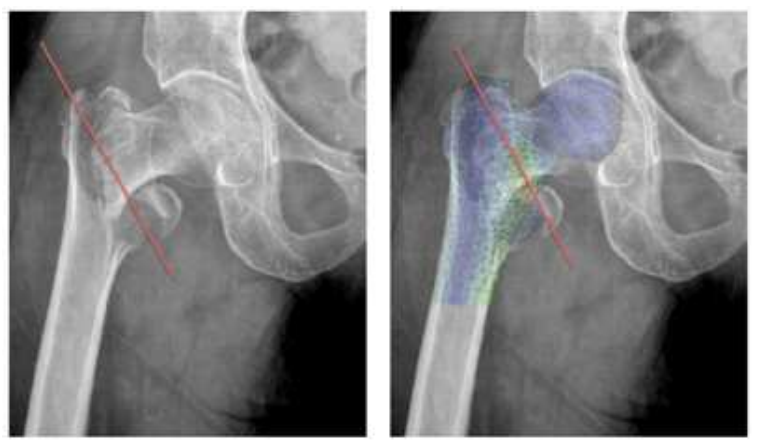

Fig. 1. Fracture plane of a pertrochanteric femur fracture (left) and FEA from Aversa et al. (2016) 
Presently, total hip replacements performed on patients younger than 65 and therefore with a higher life expectation time needing a longer durability of the orthopedic implant, do not endure for more than 15 years.

The orthopedic prostheses used today are made of metal alloys, plastics and ceramics materials with well defined properties and characteristics. In particular, due to their high biocompatibility and high mechanical strength, some metal alloys based on Titanium or Cobalt Chrome are commonly used and they represent almost all of the prosthetic components. These traditional prosthetic products are made from assessed technologies such as metal forgings or melting followed by mechanical machining. These processes do not allow the optimal design solutions for a better biomechanical bone integration. The application of other scaffolding material on the surfaces to create a more osteoinductive and osteoconducive substrate for a healthy bone in growth has to be necessarily undertaken (Schiraldi et al., 2004; Gramanzini et al., 2016). However, these processes, even if very expensive, do not provide a sufficient structural support and, often, they are not mechanically adequate. Full Metal implants, on the other hand, can allow a quick and satisfactory structural restoration of functional mobility while maintaining acceptable running costs. However, other than the high invasiveness, these implants do not represent the ideal solution, especially for younger patients who have high life expectation, both in terms of duration and biomechanical osteointegration. This first-generation of prostheses, in fact, do not technically represent the optimal structure for a good osseo and bio-mechanical integration with the bone living tissues of the area surrounding the implant.

Comparing a metal prosthesis with a human bone at a radiographic examination it is evident the difference in density and mass that can induce not healthy bone growth (Apicella et al., 2015).

Frost (1994) reports that bone adaptive properties depend on the ranges of physiological strains to which bone is subjected. Implantation of the prosthesis could than favor reabsorption processes leading to more and a more insecure and unstable condition that, until the prosthesis shows signs of weakness, will move from its location producing serious consequences and pain to the patient. In more severe cases the implant breaks down, making it necessary for its immediate replacement.

Biomechanics and Biomimetics: A Way to Foster Advanced Materials and Technologies Potentials

The implants are then expected to serve for much longer period without failure or surgical revision. The design and improvement of correct combinations of materials and prosthetic systems showing higher endurance and improved biocompatibility becomes mandatory.
The prosthetic implant replacing the resected bone should expected to ensure an "equivalent stiffness" (combination of material elastic modulus and prosthesis shape) matching that of the missing part of the bone and that of the residual osseous region where it is implanted.

Due to its orthotropicity and differently density of the structure, the elastic modulus of the bone may vary from 4 to $20 \mathrm{GPa}$, which depends on the bone type and direction of loading. The metal and ceramic implant materials adopted today, which are characterized by stiffnesses higher than those of bone where they are implanted, strongly alters the stresses physiological local distribution of the deformation with the unwanted bone reabsorption around the implant and, consequently, with an increase of the risk of implant failure.

This biomechanical mismatching leads to the lowering of the biological strain level needed for healthy bone growth (Frost, 1994) and to the loss of the bone mass due to this low level of loading know as stress shielding.

A customized material with improved combination of the needed strength and stiffness that match that of the bone has to be used for biomechanical integration of the implant. This higher biomechanical compatibility will avoid implant loosening and will increase the its service lifespan avoiding additional surgery for revision and permitting a good bio-integration (bone growth).

Innovative biomimetic materials for tissue engineering that are based on hydrophilic polymers have been developed by our research group and have shown attractive physical, biological and mechanical properties for applications in the biomedical field (Schiraldi et al., 2004). Highly biocompatible novel hybrid scaffolding materials based on fumed silica and hydrophilic poly-(Hydroxyl-Ethyl-Methacrylate) (pHEMA) to be used in concurrence to metal prostheses have been developed by the Authors.

The structural metal scaffold of the new prostheses will be made of Titanium alloys using the additive technology based on the principle of melting thin layers of Titanium powder (about 50 microns) one over the other until the achievement of the desired component is completed (see lower right of Fig. 9). Then the nanostructured and osteoconductive biomimetic material developed in our previous studies (Schiraldi et al., 2004) can cover the Titanium structural prosthetic scaffold.

These biological hybrid prostheses, which are made using synthetic materials able to induce the growth of biological networks and a metal structural scaffold, may favor the birth of new classes of hybrids orthopedic solutions in the medical field (Huiskes et al., 1987).

The new hybrid bio-prosthesis could drastically reduce the stress shielding phenomena while providing an advantageous improvement of prosthesis lifetime compared to traditional solutions. The recovery of optimal joint functionality will lead to the improvement of the quality of life of the patient, who perceives significantly abating the risk of new surgical operation. 
The requirement of envisaging the potential structural alteration that can be induces by the inadequate use of not biologically compatible prostheses in the structure and morphology of the bone, has forced our studies in the direction of the assessment of faithful models of the femur that could account for real bone type distribution and to its orthotropic behavior (Aversa et al., 2016).

The development of faithful models has already enabled us to prepare prostheses that could restore the natural physiological stresses and strains patterns in the bone (Apicella et al., 2010; Gramanzini et al., 2016; Perillo et al., 2010; Sorrentino et al., 2007; 2009).

In this study a femur FEM model, which has been developed in a previous work to correctly represent structural behavior of the femur head (Aversa et al., 2016), has been analyzed for the stress and strains distribution over the entire bone stem and head and it has been modified to account for femur head resection and substitution with a Titanium hip joint prosthesis. A comparison between the biological stress and strain distributions in the femur models of the sound and prothesized femurs could help in the understanding of the correct design procedures needed to design new innovative biomimetic prostheses.

\section{Materials and Methods}

Medical Image Segmentation has been derived from CT using the Mimics software (Materialise, Belgium) to process a patient medical image. As reported in Fig. 2, processing of CT resulted in a highly accurate 3D solid model of the patient pelvis and femur anatomy.

New prosthetic engineering applications through a combined use of Mimics and 3-Matic (Materialise, Belgium) software's could be derived from the study of the biomechanics of these bone districts.

A 3D solid and Finite Element Models (FEM) have been developed in a previous work to simulate the external and internal morphology of the femur as already done for other complex bone structures accounting for the orientation and densities of the head trabecular systems (Aversa et al., 2016; 2009; Apicella et al., 2010; Beaupre and Hayes, 1985; Reilly and Burstein, 1974; 1975). The procedure is illustrated in the Fig. 3-6. The external geometry of femur and portion of the pelvis have been reconstructed by generating a three-dimensional volume that interpolates the CT scans (Fig. 3).

The results were then imported in the 3 Matic software for surface and solid meshing optimization, Finite element model preparation and material properties definition (Aversa et al., 2016; Apicella et al., 2010; 2015).

The results of tethraedric element material distribution are shown in Fig. 4 (the yellows correspond to different stiffnesses cortical bone mechanical properties while greens are relative the trabecular bone of different densities).

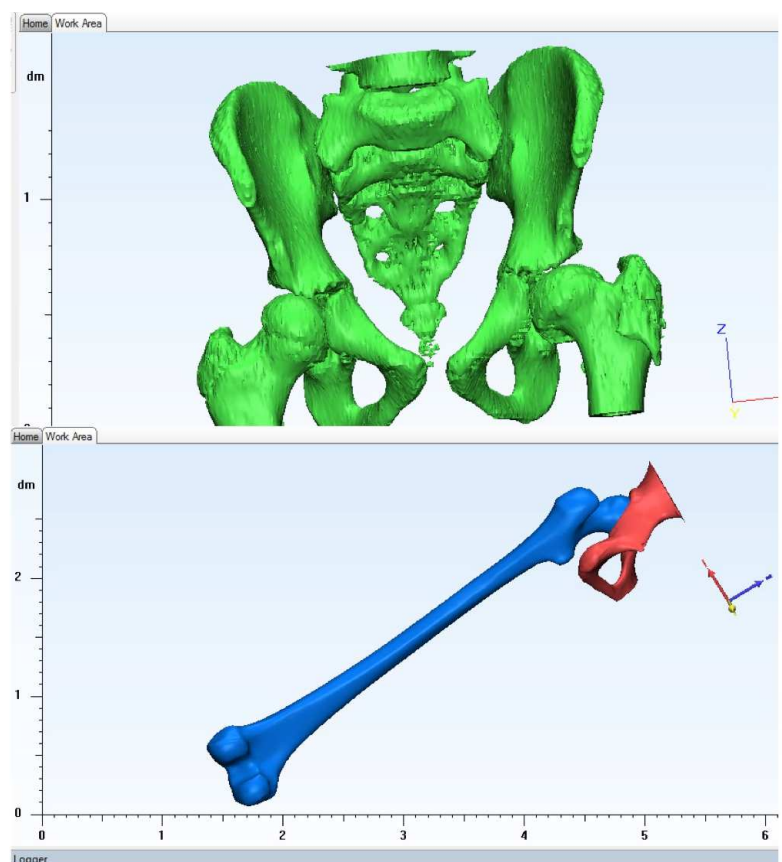

Fig. 2. Biofidel medical Image Segmentation of a patient pelvis and femur joint

The same procedure was applied to the preparation of the Finite Element Analysis (FEA) model of the femur with the resection of the head, which has been virtually done at the fracture plane reported in Fig. 1 and for the Titanium traditional prosthesis.

Internal modeling of the resected femur and the of the meta prosthesis have been realized by defining threedimensional internal tethraedric meshing distribution and size optimization, as indicated in the upper part of Fig. 5 and 6 , respectively.

The solid mesh elements of the resected femur have been sequentially associated to the patient bone densities according to the Hounsfield (HU) scale. This scale linearly quantifies the X-rays attenuation coefficients in the tissues, assigning, by using the Mimics software, the proper elastic moduli to the corresponding elements of the FEM model (lower part of Fig. 5).

The meshed solid models of the resected femur and of the $\mathrm{Ti}$ prosthesis were assembled accounting to the correct positioning of the implant using the Mimics software (Fig. 7).

New remeshing of the tethraedric elements were run in 3Matic (Fig. 8) searching for the node congruency at the bone-implant interfaces.

The mechanical properties have been assigned considering the cortical and trabecular bone characteristics.

In particular, the systems have been considered as isotropic materials and the mechanical properties have been coupled with each single tethraedric element characterized by equivalent Hounsfield (HU) densities scale. The elastic and shear moduli of the trabecular bone were derived as a fraction of those of the cortical 
bone cortical bone with a direct proportionality with the porosity measured by the axial tomography. In this scale, the value of the fat is about -110 , the muscles around 40 , the trabecular bone stay between 100 and
300 while the cortical one covers the values between the high density cortical bone (300) and about 2000, that matched in our bone systems to Elastic moduli ranging from 0,87 to $15.0 \mathrm{GPa}$.

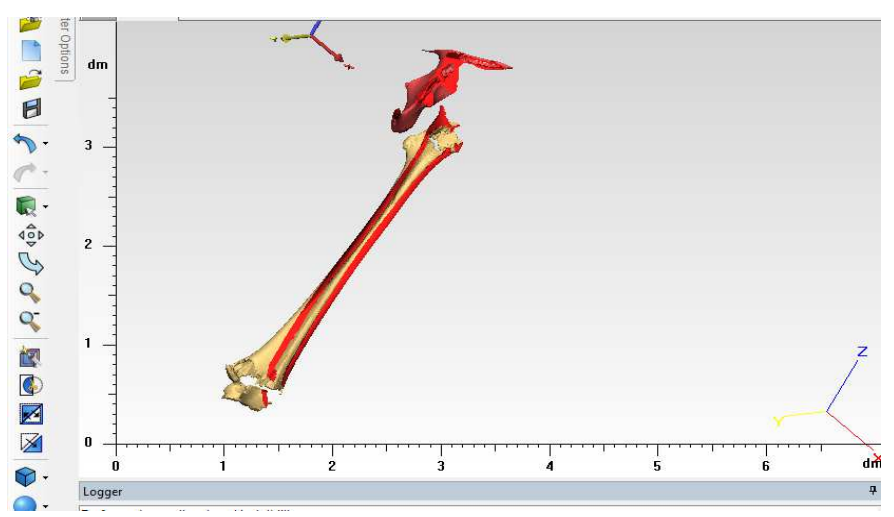

Fig. 3. Biofidel 3D solid modelling of a patient femur
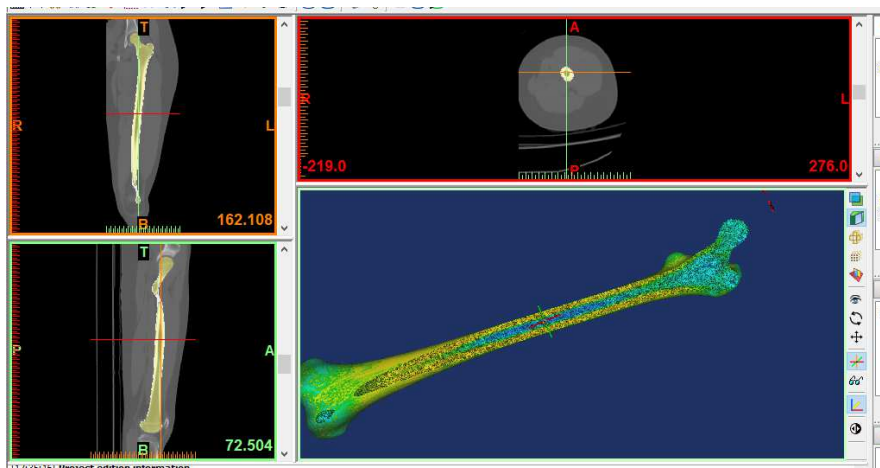

Fig. 4. Surface meshing optimization of the biofidel patient entire (not resected) femur model (Aversa et al 2016)
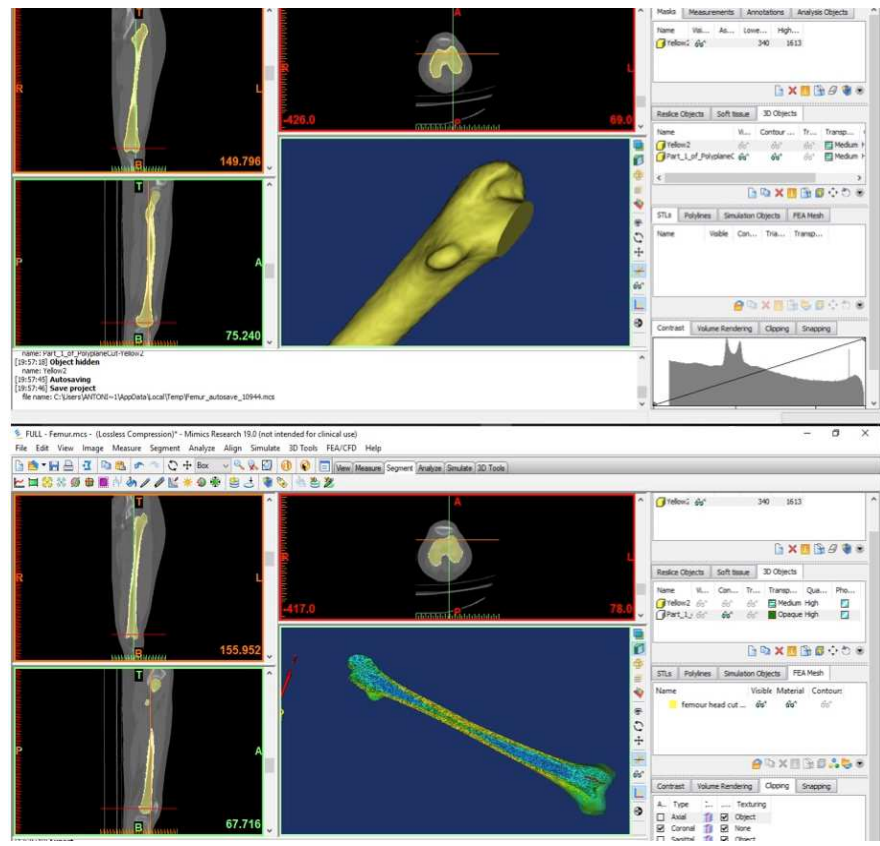

Fig. 5. 3D meshing optimization of the biofidel patient femur model 


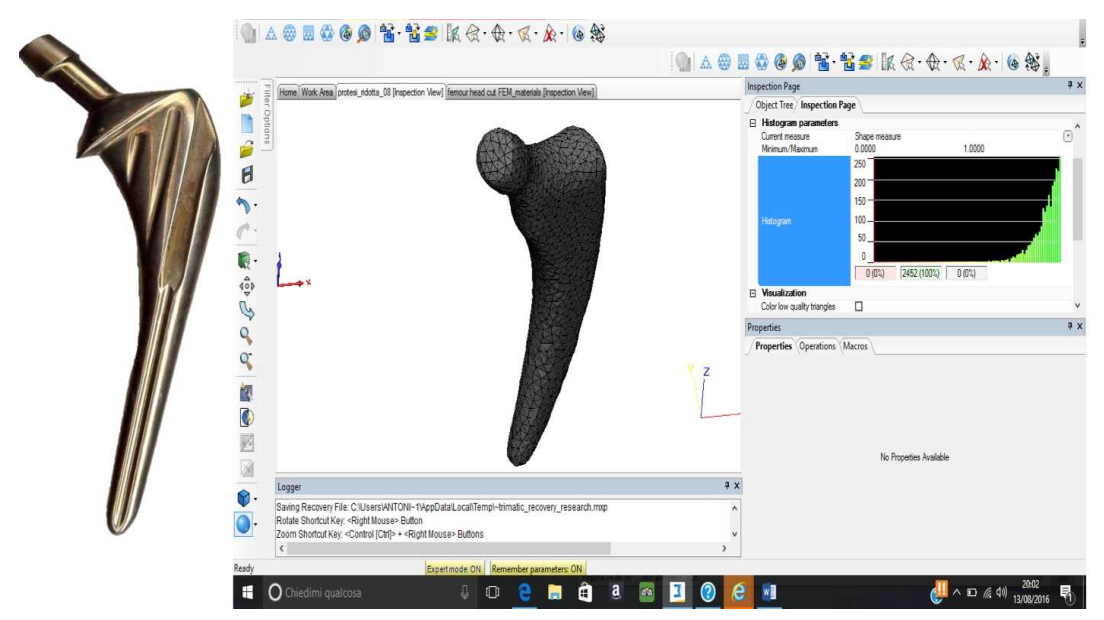

Fig. 6. 3D meshing optimization of a Titanium traditional prosthesis

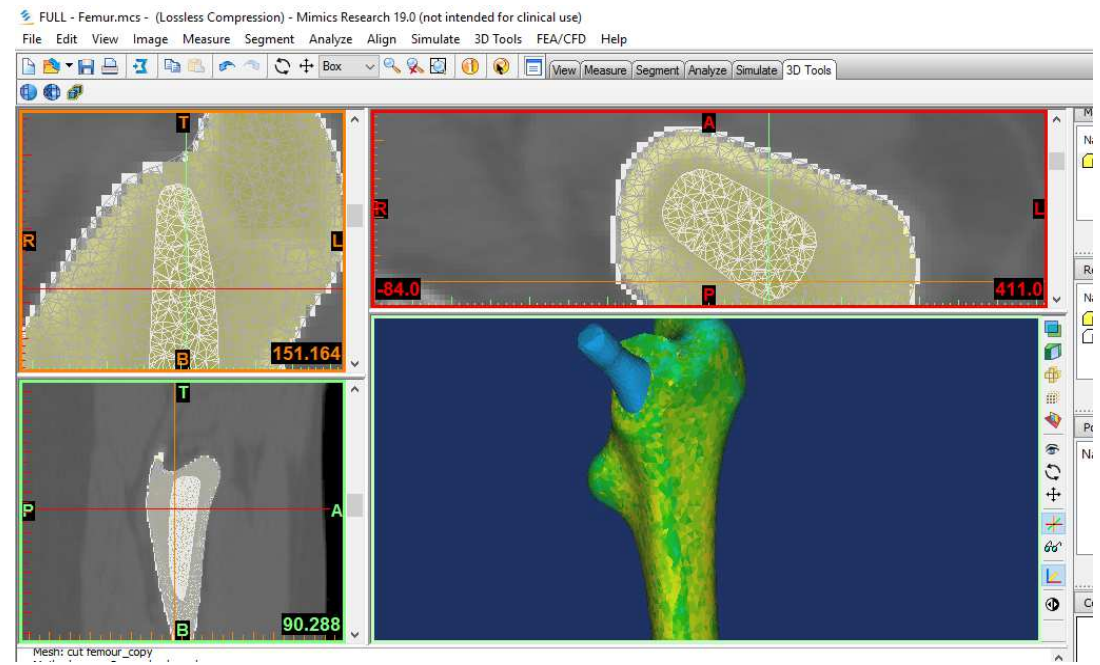

Fig. 7. Assembly of the FEM model for element congruency and material properties definition (Green, trabecular bone, Yellow, cortical bone, Blue, Ti

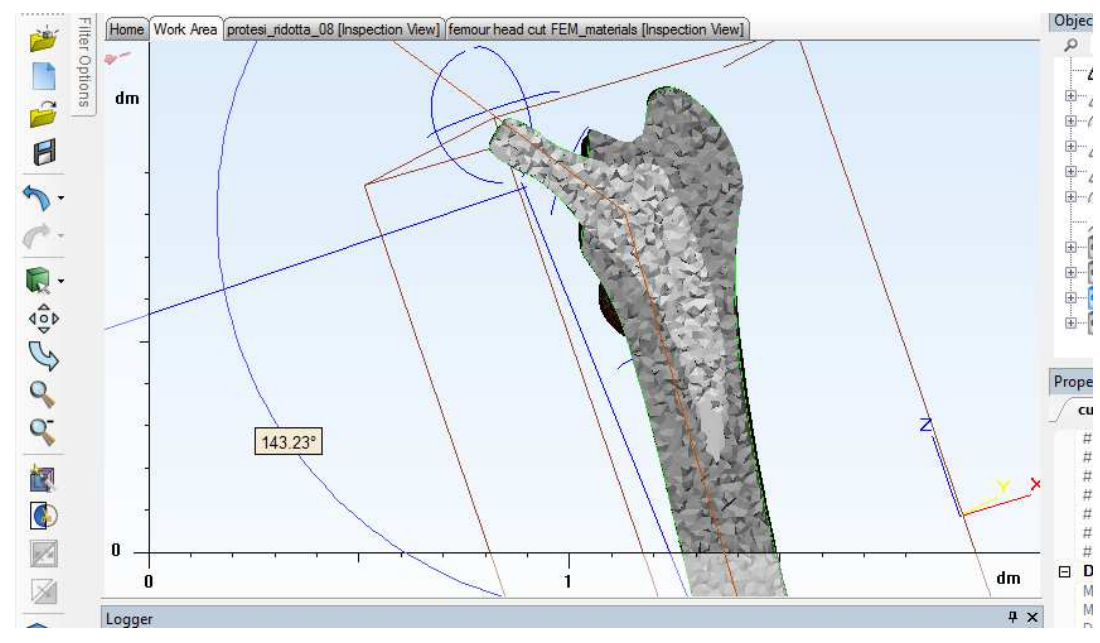

Fig. 8. Assembly of the resected femur and Titanium rigid hip prosthesis 
The solid mesh elements of the trabecular-oriented material have been assigned by operating on the internal structure accounting for the real bone trabecular morphology that we found in the proximal end, (Fig. 6) as indicated in Aversa et al. (2016).

\section{Variable rigidity Ti prostheses Design}

In order to avoid excessive stress shielding effects on the resected femur diaphysis (shaft), trabecular hip joint prosthesis has been properly designed according to the characteristic and specific rigidity of each diaphysis section interested by the prothesization.

The mechanistic model of the hip proximal epiphysis proposed by Kummer (1986) has been transferred to the hip joint prosthesis design where the presence of isostatic lines that characterizes the oriented trabecular systems, is reported in Fig. 9 (left hand side).

Moreover, biomimetic prosthesis should present rigidities along the isostatic lines that match those of the bone where it is placed.

Five regions of the prosthesis have been chosen to assign differentiated decreasing rigidities (right hand side of Fig. 9).

The state stress acting on the system and the observation of the isostatic lines described by Kummer (1986) has been used to define the morphology of the porosity characterizing the trabecular structure of the stem and head of the prosthesis in different zones.

These regions should be characterized by different rigidities that progressively decrease from the highly rigid head (region 1 in Fig. 9) down to the highly flexible (region 5 in Fig. 9).

The isostatic lines and the morphology differences of the isorigidity trabecular regions are better appreciated by comparing trabecular structure of isostatic and isorigidity regions (Fig. 10b and 10c).

The diverse orientation and shape of the Titanium trabecular porosity is evident in the internal structure reported in the Fig. $10 \mathrm{~b}$ and $\mathrm{c}$.

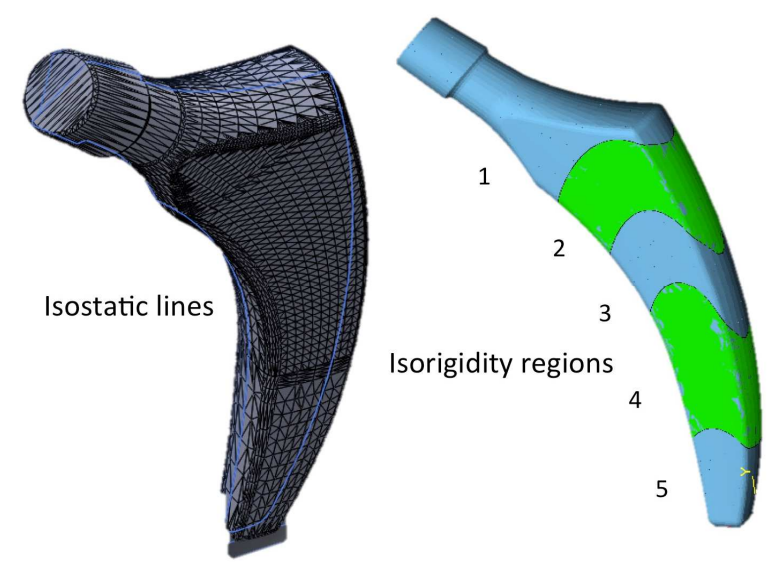

Fig. 9. Left, Isostatic lines (Kummer, 1986) and right, Isorigidity regions chosen for the biomimetic prosthesis trabecular structure design
An example of isorigidity trabecular structure obtained by Ti alloy powder sintering by Electron Beam is reported in Fig. 10d. The apparent elastic moduli of the trabecular structures in the isorigidity regions vary from $20 \mathrm{GPa}$ for region 1 to $11,8,4$ and $1 \mathrm{GPa}$ for regions 2, 3, 4 and 5, respectively.

The orientation of trabecular system observed in the lower left of Fig. 10b, which is observed in the area of the prosthesis stem corresponding to the femur epiphysis subjected to tensile stresses, would preserve and provide the requested continuity of the rigidity in each specific region reported in Fig. 10a.

The previously CT computed values of bone densities have been then related to the isostatic lines of cortical and trabecular bone of the sound and resected femur epiphysis by considering each tethraedric mesh element oriented along the stress isostatic directions observed in the femur proximal epiphysis. Analogously, the same criterion has been applied (isostatic line orientation) for the biomimetic trabecular Titanium prosthesis material definition.

\section{FEM Analysis: Sound and Prothesized Femur, with Traditional Rigid and New Flexible Ti Prostheses}

On the basis of the $3 \mathrm{D}$ models of the sound femur and the resected femurs prosthesized with rigid and flexible stem prosthesis, a structural evaluation under the same loading conditions has been developed and the results critically compared (Rohlmann et al., 1982).

The characteristic biometric parameters of the patient femur-hip system have been carried out before running the structural analysis. The 3Matic software has been used to identify the direction of the loading axis, the center of the proximal epiphysis head sphere and the center of the joint epicondyle and mechanical axis of rotation of the knee. Namely, the following parameters have been measured: The mechanical axis between the centers of the proximal and distal epiphyses, the angle of $143.40^{\circ}$ between the femoral neck and diaphysis axes and the divergence of $36.65^{\circ}$ between the neck axis with the axis of epicondyles (Taylor et al., 2007; Weinans et al., 1992).

Finally (before running the FEM structural analyses) the physiological loads and constrains have been evaluated.

\section{Loading Condition and Imposed Constrains}

The equilibrium monopodalic posture has been considered as an extreme severe loading condition with a rotation around the center of hip joint where the gluteus muscular force equilibrates the moment imparted by the body weight force. Considering the weight of the patient of $100 \mathrm{Kg}$, the gluteus muscular force that is applied to the great trochanter is of $1800 \mathrm{~N}$ and the hip joint reaction force is $2740 \mathrm{~N}$ (these values have been calculated using the biometric data of Fig. 11). 


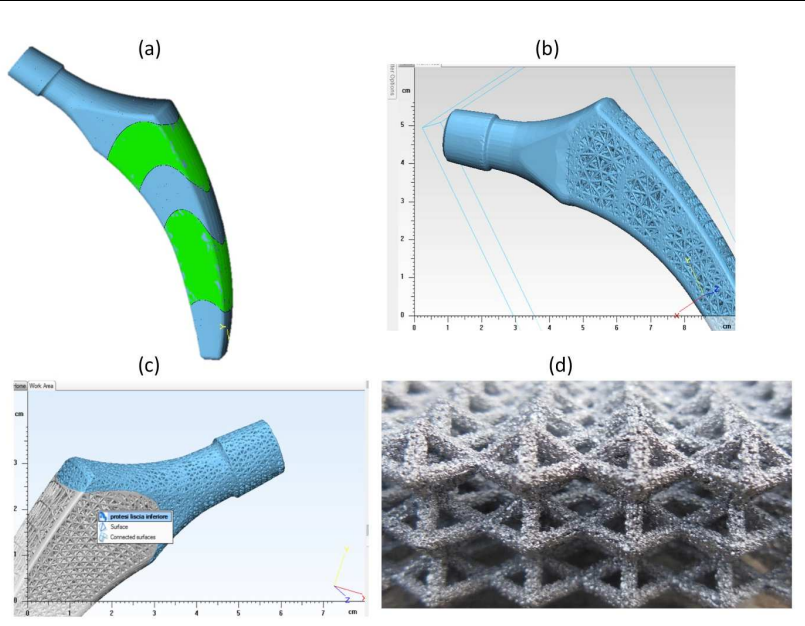

Fig. 10. Flexible trabecular hip joint prosthesis with different stem and head rigidities and orientations. (a) region of equivalent stiffness, (b) the overall flexible prosthesis, (c) internal trabecular structure, (d) Orthotropic trabecular structure obtain

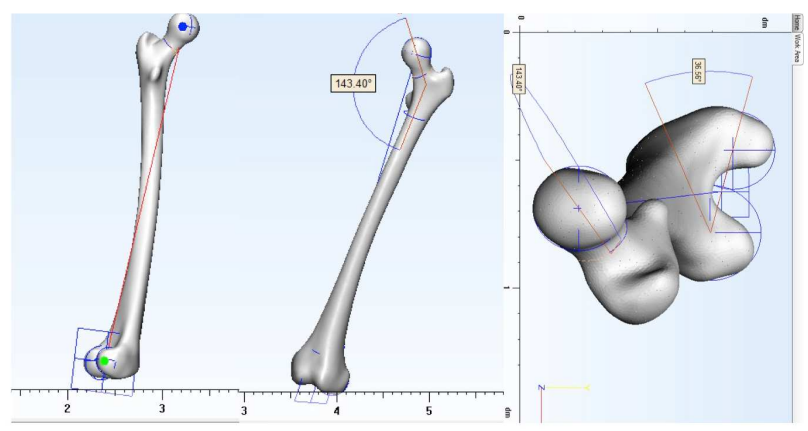

Fig. 11. Biometric analysis (Aversa et al., 2016): Mechanical axis of the femur (left); Angle of the femoral neck $\left(143.40^{\circ}\right.$, Center); Angle of divergence of the neck with the axis of epicondyles $\left(36.65^{\circ}\right.$, Right)

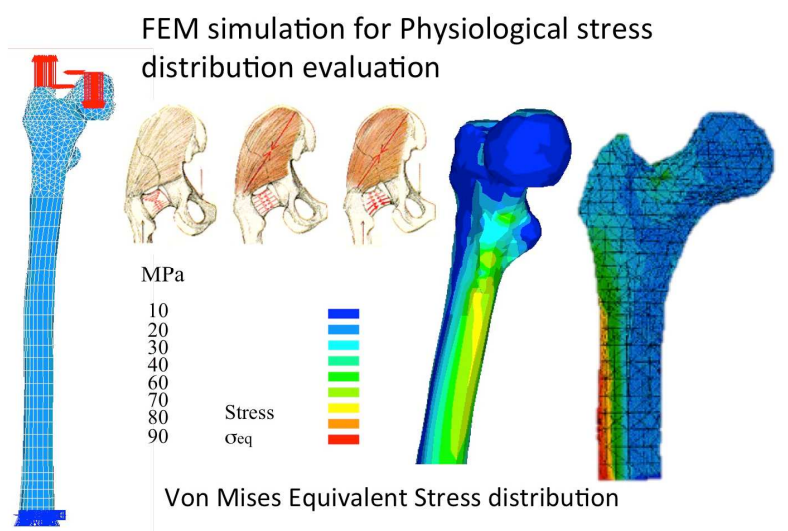

Fig. 12. Phisiological Equivalent Von Mises stresses in the femur from our biofidel Finite Element Model (Aversa et al., 2016)

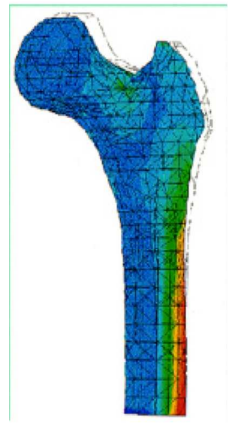

(a)

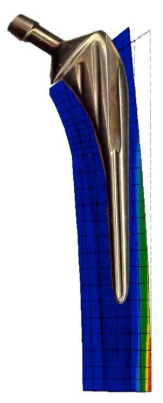

(b)

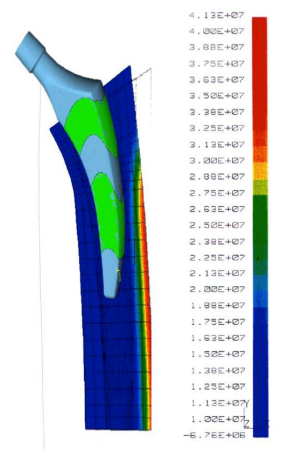

(c)
Fig. 13. Equivalent Von Mises stresses distribution in the proximal femur epiphysis for (a) sound femur, (b) rigid $\mathrm{Ti}$ prosthesis, and (c) flexible trabecular prosthesis

The reaction force of the Gluteus muscle has been uniformly distributed around 100 nodes of great trochanter FEM model area, while reaction force in the hip joint has been spread over 50 nodes of the femur head (see upper left in Fig. 12).

\section{Results and Discussion}

In this study we defined the procedure to prepapre a biofidel model the femur able to correctly describe its biomechanical structural behavior. Von Mises strain criterion has been used to compare and validate the new designed trabecular flexible prostheses. Von Mises is an energetic criterion that can properly quantify the ability of the bone to withstand high loads (Fig. 12).

The different structural behavior of the two models is also makes evident by the Von Mises stress distribution, clearly different at the posterior and the front level (right hand end of Fig. 12 and 13a). Posteriorly, strain distribution confirms the presence of bending effects, with the highest values homogeneously distributed in the anatomical diaphysis regions (Fig. 13a).

Figure 13 clearly shows the significant modification of the stress distribution in the sound femur (Fig. 13a), the resected femur with the rigid Ti prosthesis (Fig. 13b) and with the flexible stem prosthesis (Fig. 13c). As already specified in a previous paper, the rigidly prosthesized femur misses the physiological flexural stresses distribution of a sound diaphysis (Fig. 13a) leading to a stress concentration only in the medial region a "stress shielded" proximal (due to the stem high rigidity that does not allow diaphysis physiological flexure).

The absence of stress (and related strains state) could induce, over the time, significant bone structure modification (bone reabsorption).

Conversely, the biomimetic flexible trabecular prosthesis model, realizes a more uniform strains distribution (Fig. 13c) that better mimics in terms of 
stress distribution and intensities the action of the load on a sound femur (Fig. 13a).

\section{Conclusion}

The innovative aspects of our work resides in the fact that, contrariwise to the currently used prosthesis that are only designed for the replacement of a damaged hip joint, the proposed biological hybrid prostheses are able to be completely biomimetic since mimicking biological stress distribution that stimulates physiological tissues regeneration.

The average life of a prosthesis today is about 10/15 years, while the new "biomimetic prosthesis" will have a longer life that can be estimated to be over 20/25 years.

This aspect is very important because the average human lifespan is progressively increasing and this consequently increases the number of orthopedic surgical operation and social and health care related costs.

The design and fabrication of customized different densities trabecular porous structures of innovative "hip joint biomimetic prosthetic systems", which could better integrate with the physiological biomechanics of the femur where they are implanted, is then possible by correctly using such biofidel models.

The aim of the investigation presented here is to create added value by combining the existing research on biomechanical results with innovative prosthesis design and structural simulation activities.

Transfer results across academic and industrial research on biomechanics and clinical studies lead to an acceleration of the innovation and creation of the profit while improving the quality of life of the patients with prostheses.

This work identifies a number of design criteria to foster the potential to enable new medical therapies contributing to customized health care, to create and improve the technological basis and to increase the resource efficiency in the context of industrial and manufacturing processes.

The new type of biomimetic implants can find applications in orthopedics of knee, ankle, hip, shoulder and spine.

A further field of application of the product is surgical oncology to support and facilitate bone regeneration resulting in massive losses due to interventions of removal of both primitive and metastatic tumors.

The prosthetic system might allow a better functional recovery by promoting bone recreation to ensure a good hold of the load, even going to impact on the quality of life of the individual patient, already seriously compromised by the basic oncological pathology.

The concept of combining a metal support structure (to guarantee the resistance to the load) with a biomimetic scaffold (which promotes regeneration) finds application in all those sectors of the therapeutic surgery involving the removal of bone and requiring a stimulation of the regeneration of resected tissue.

\section{Acknowledgement}

We acknowledge and thank $\mathrm{Mr}$ Taher M. AbuLebdeh, Associate Prof at North Carolina A and T State Univesity, United States and Mr Muftah H. El-Naas PhD MCIC FICCE QAFCO Chair Professor in Chemical Process Engineering Gas Processing Center College of Engineering Qatar University and Ms Shweta Agarwala, Senior Research Scientist at Singapore Center for 3D Printing Nanyang Technological University Singapore for their suggestions and comments.

\section{Funding Information}

This research was partially funded by Italian Ministry of University and Research with the project FIRB "Future in Research 2008", \# RBFR08T83J.

\section{Author's Contributions}

All the authors contributed equally to prepare, develop and carry out this work.

\section{Ethics}

This article is original. Author declares that are not ethical issues that may arise after the publication of this manuscript.

\section{References}

Annunziata, M., R. Aversa, A. Apicella, A. Annunziata and D. Apicella et al., 2006. In vitro biological response to a light-cured composite when used for cementation of composite inlays. Dental Mater., 22: 1081-1085. DOI: 10.1016/j.dental.2005.08.009

Apicella, D., R. Aversa, E. Ferro, D, Ianniello and A. Apicella, 2010. The importance of cortical bone orthotropicity, maximum stiffness direction and thickness on the reliability of mandible numerical models. J. Biomed. Mater. Res. Part B Applied Biomater., 93: 150-163. DOI: 10.1002/jbm.b.31569

Apicella, D., M. Veltri, P. Balleri, A. Apicella and M. Ferrari, 2011. Influence of abutment material on the fracture strength and failure modes of abutmentfixture assemblies when loaded in a bio-faithful simulation. Clin. Oral Implants Res., 22: 182-188. DOI: $10.1111 /$ j.1600-0501.2010.01979.x

Apicella, D., R. Aversa, M. Tatullo, M. Simeone and S. Sayed et al., 2015. Direct restoration modalities of fractured central maxillary incisors: A multi-levels validated finite elements analysis with in vivo strain measurements. Dental Mater., 31: e289-e305. DOI: $10.1016 /$ j.dental.2015.09.016 
Ashman, R.B., S.C. Cowin, W.C. Van Buskirk and J.C. Rice, 1984. A continuous wave technique for the measurement of the elastic properties of cortical bone. J. Biomechan., 17: 349-361.

DOI: 10.1016/0021-9290(84)90029-0

Ashman, R.B. and J.Y. Rho, 1988. Elastic modulus of trabecular bone material. J. Biomechan., 21: 177-81. DOI: 10.1016/0021-9290(88)90167-4

Aversa, R., D. Apicella, L. Perillo, R. Sorrentino and F. Zarone et al., 2009. Non-linear elastic threedimensional finite element analysis on the effect of endocrown material rigidity on alveolar bone remodeling process. Dental Mater., 25: 678- 690. DOI: $10.1016 /$ j.dental.2008.10.015

Aversa, R., F.I.T. Petrescu, R.V.V. Petrescu and A. Apicella, 2016. Biomimetic finite element analysis bone modeling for customized hybrid biological prostheses development. Am. J. Applied Sci., 13: 1060-1067. DOI: 10.3844/ofsp.10904

Beaupre, G.S. and W.C. Hayes, 1985. Finite element analysis of a three-dimensional open-celled model for trabecular bone. J. Biomech. Eng., 107: 249-56. DOI: $10.1115 / 1.3138550$

Burnstein, A., D.T. Reilly and M. Martens, 1976. Aging of bone tissue: Mechanical properties. J. Bone Joint Surgery, 58: 82-86. PMID: 1249116

Carter, D.R. and W.C. Hayes, 1977. The compressive behavior of bone as a two-phase porous structure. J. Bone Joint Surgery, 59A: 954-962. PMID: 561786

Dalstyra, M., R. Huiskes, A. Odgaard and L. Van Erning, 1993. Mechanical and textural properties of pelvic trabecular bone. J. Biomechan., 26: 349-361. PMID: 8478354

Frost, H.M., 1994. Wolff's law and bone's structural adaptations to mechanical usage: An overview for clinicians. Angle Orthod., 64: 175-188. PMID: 8060014

Gramanzini, M., S. Gargiulo, F. Zarone, R. Megna and A. Apicella et al., 2016. Combined microcomputed tomography, biomechanical and histomorphometric analysis of the peri-implant bone: A pilot study in minipig model. Dental Mater., 32: 794-806.

DOI: 10.1016/j.dental.2016.03.025

Huiskes, R., H. Weinans, H.J. Grootenboer, M. Dalstra and B. Fudula et al., 1987. Adaptive boneremodeling theory applied to prosthetic-design analysis. J. Biomech., 20: 1135-1150.

DOI: 10.1016/0021-9290(87)90030-3

Kummer, B., 1986. Biomechanische Grundlagen der Statik des Hüftgelenks. Zeitschrift Orthop. Grenzgebiete, 124: 179-187.

DOI: $10.1055 / \mathrm{s}-2008-1044544$

Mullender, M.G. and R. Huiskes, 1995. Proposal for the regulatory mechanism of Wolff's law. J. Orthop. Res., 13: 503-512. DOI: 10.1002/jor.1100130405
Oh, I. and W.H. Harris, 1976. Proximal strain distribution in the loaded femur. An in vitro comparison of the distributions in the intact femur and after insertion of different hip-replacement femoral components. J. Bone Joint Surgery, 60: 75-85. PMID: 624762

Perillo, L. R. Sorrentino, D. Apicella, A. Quaranta and E. Gherlone et al., 2010. Nonlinear visco-elastic finite element analysis of porcelain veneers: A submodelling approach to strain and stress distributions in adhesive and resin cement. J. Adhesive Dent., 12: 403-413.

Reilly, D.T. and A.H. Burstein, 1974. The mechanical properties of cortical bone. J. Bone Joint Surgery, 56: 1001-1021.

Reilly, D.T. and A.H. Burnestain, 1975. The elastic and ultimate properties of compact bone tissue. J. Biomechan., 8: 393-405. DOI: 10.1016/0021-9290(75)90075-5

Rohlmann, A., U. Mossner, G. Bergmann and R. Kolbel, 1982. Finite-element-analysis and experimental investigation of stresses in a femur. J. Biomed. Eng., 4: 241-246. DOI: 10.1016/0141-5425(82)90009-7

Schiraldi, C., A. D'Agostino, A. Oliva, F. Flamma and A. De Rosa et al., 2004. Development of hybrid materials based on hydroxyethylmethacrylate as supports for improving cell adhesion and proliferation, Biomaterials, 25: 3645-3653. DOI: $10.1016 /$ j.biomaterials.2003.10.059

Sorrentino, R., R. Aversa, V. Ferro, T. Auriemma and F. Zarone et al., 2007. Three-dimensional finite element analysis of strain and stress distributions in endodontically treated maxillary central incisors restored with diferent post, core and crown materials. Dent. Mater., 23: 983-993.

DOI: 10.1016/j.dental.2006.08.006

Sorrentino, R., D. Apicella, C. Riccio, E. Gherlone and F. Zarone et al., 2009. Nonlinear visco-elastic finite element analysis of different porcelain veneers configuration. J. Biomed. Mater. Res. Part B Applied Biomater., 91: 727-736. DOI: 10.1002/jbm.b.31449

Gottesman, T. and Z. Hashin, 1980. Analysis of viscoelastic behaviour of bones on the basis of microstructure. J. Biomechan., 13: 89-96. DOI: 10.1016/0021-9290(80)90182-7

Taylor, D., J.G. Hazenberg and T.C. Lee, 2007. Living with cracks: Damage and repair in human bone. Nat. Mater., 6: 263-268. DOI: 10.1038/nmat1866

Weinans, H., R. Huiskes and H.J. Grootenboer, 1992. The behavior of adaptive bone-remodeling simulation models. J. Biomech., 25: 1425-1441. DOI: 10.1016/0021-9290(92)90056-7 